Research Journal of Pharmacology 13 (3): 35-41, 2019

ISSN: $1815-9362$

(C) Medwell Journals, 2019

\title{
Radio-Pathological Correlation in Patients over 18 Years Old Who Present Renal Tumors Attended at the Oncological Institute Dr. Juan Tanca Marengo, Solca-Guayaquil in the Period 2014-2016
}

\author{
${ }^{1}$ Dayanara Ordonez Calderon, ${ }^{1}$ Naomi Ordonez Calderon, ${ }^{1}$ Fuad Huaman Garaicoa, \\ ${ }^{1}$ Marco Ordonez Marin, ${ }^{2}$ Carlos Jacome Pilco, ${ }^{2}$ Darwin Nunez, \\ ${ }^{2}$ Rivelino Ramon and ${ }^{3}$ Diego Moposita \\ ${ }^{1}$ Facultad De Ciencias Medicas, Universidad Catolica Santiago De Guayaquil, Carrera De Medicina, \\ Av. Pdte. Carlos Julio Arosemena Tola, Cp: 09061, Guayaquil, Ecuador \\ ${ }^{2}$ Facultad De Ciencias Agropecuarias, Universidad Estatal De Bolivar, \\ Recursos Naturales Y Del Ambiente, Cp: 020150, Guaranda, Ecuador \\ ${ }^{3}$ Escuela De Ingenieria Agroindustrial, Universidad Nacional De Chimborazo, \\ 060110 Riobamba, Ecuador
}

\begin{abstract}
Renal cell carcinoma is currently a tumor with an increasing incidence, associated with a higher mortality rate with an equal proportion for both sexes. After 59 years of age, there is an increased risk of kidney cancer as the incidence increases with age. In most cases renal cell carcinoma is discovered incidentally by independent imaging findings in renal pathology and in symptomatic cases the clinical triad consisting of macroscopic hematuria, lumbar fossa pain and presence of palpable mass. Renal clear cell carcinoma was the most frequent histological type with a grade 2 Fuhrman present in the majority of cases. It has been shown that renal tumors can infiltrate the renal capsule, perirenal fat, Gerota's fascia, renal sinus, renal vein, vena cava and the lymphovascular system. The presence of tumor necrosis is associated with a poor prognosis in patients with renal masses $>7 \mathrm{~cm}$. The mean tumor size was $7.17(2-20) \mathrm{cm}$. The lymph node metastasis is very frequent, being the liver, lungs and bones the anatomical areas more prone to metastasize. The majority of patients diagnosed are in stage 1 and 3 . The degree of surgical complexity and the surgical procedure indicate that there is no correlation $(\mathrm{p}=0.00)$ between the imaging report and the surgical evaluation.
\end{abstract}

Key words: Renal tumors, renal cell carcinoma, diagnosis of computed tomography and anatomopathological diagnosis, surgical procedure, kidney cancer, symptomatic cases

\section{INTRODUCTION}

Renal tumors are abnormal cell growths that affect the renal parenchyma. These can be benign or malignant according to, their cell typology. Benign tumors are those tumor lesions that do not invade adjacent normal tissue. In contrast, malignant tumors have the ability to invade and damage tissues near the tumor and in turn spread throughout the body. It represents the third most frequent urological tumor in men and the second malignant tumor in women (Villaverde et al., 2017).

Epidemiology: From 1975-2015, the incidence of kidney cancer increased in countries like the United States, from 7-16 cases per 100,000 inhabitants. Currently, kidney and renal pelvis cancers are the sixth most common cancer in men in the US (Villaverde et al., 2017).
In most of Europe, the incidence and mortality from kidney cancer has decreased in the last decade. Renal cell carcinoma is more common in people of Northern European and North American descent than in those of Asian or African descent. The median age at the time of diagnosis is 64 years (Karam et al., 2011).

Worldwide, renal masses are not very frequent, only represent $3 \%$ of adult renal tumors and within these $90-95 \%$ are renal cell carcinoma. With an age range of 50-70 years. At the American level, renal tumors are more frequent in men than in women, representing 5\% in men and only 3\% in women (Byun et al., 2016; Pilskog et al., 2017).

The majority of renal masses are asymptomatic and non-palpable. Currently $50 \%$ of all patients with renal cell tumor have been evidenced the problem by means of incidental image studies and $35 \%$ of them present

Corresponding Author: Dayanara Ordonez Calderon, Facultad De Ciencias Medicas, Universidad Catolica Santiago De Guayaquil, Carrera De Medicina, Av. Pdte. Carlos Julio Arosemena Tola, Cp: 09061, Guayaquil, Ecuador 
(a)

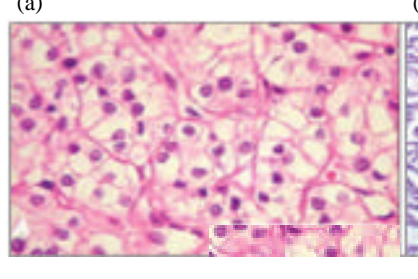

(d)

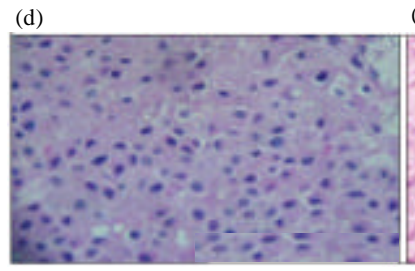

(b)

(c)

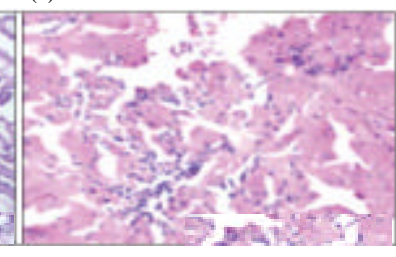

(e)

(f)

Fig. 1: Cellular histology of kidney cancer: a) Clear cells; b) Type 1 papillary; c) Type 2 papillary; d) Chromophobe; e) Collecting ducts and f) Medullary

symptoms such as: pain in the renal fossa, macroscopic hematuria and palpable abdominal mass (Borghesi et al., 2015). According to, the WHO/PAHO report in Ecuador clear cell carcinoma represents $75 \%$ of the cases, followed by chromophobe cell carcinoma in $15 \%$. Within benign neoplasms, the renal adenoma is the most frequent with $75 \%$, followed by oncocytoma in 3-7 and 20\% angiomyolipoma.

Clear cell carcinoma: The clear RCC cell originates in the epithelium of the proximal convoluted tubules and presents a predominantly expansive growth pattern. Macroscopically, it is a solid, yellowish lesion with varying degrees of internal necrosis, hemorrhage and cystic degeneration.

Papillary carcinoma: The papillary variant of this tumor is the second most common subtype of histological CRC, representing approximately $10 \%$ of cases. So, this variant presents two subtypes according to, the histological aspect and biological behavior of the lesion. The pRCC type 1 (basophil) has a single layer of basophilic cells surrounding the basement membrane, these small cells have a clear cytoplasm and a sparse hyperchromatic nucleus. In turn, the histological subtype 2 (eosinophilic) is presented with papillae covered by cells with abundant granular eosinophilic cytoplasm with prominent nucleoli associated with areas of necrosis. The pRCC type 1 is usually detected at earlier stages and is low grade than pRCC type 2 . In contrast, pRCC type 2 behaves like a high grade tumor, frequently associated with ganglionic metastases and in some cases with venous invasion (Sircar et al., 2013).
Chromophobe renal cell carcinoma: Chromophobe RCC represents $5 \%$ of all malignant renal epithelial tumors and is more frequent at 60 years such a subtype is less aggressive than clear RCC. Metastasis occurs in 7\% of cases. At the histological level, these tumors present large pale cells with reticulated cytoplasm and perinuclear halos (Sircar et al., 2013) (Fig. 1).

Clear cell RCC: Cells with lipid-rich ample cytoplasm, hence, the name of the neoplasm.

Type 1 papillary RCC: Small basophilic cells with scarce cytoplasm, organized in a spindle-shaped pattern in a single layer of cells surrounding the basal membrane.

Type 2 papillary RCC: Cells organized in a spindle-shaped pattern with papillae covered by cells with abundant eosinophilic granular cytoplasm with prominent nucleoli.

Chromophobe RCC: Large pale cells with reticulated cytoplasm and perinuclear halos.

Collecting duct RCC: Histology shows an irregular, infiltrating cells arrangement in the collecting duct walls, showing remarkable desmoplasia.

Medullary RCC: It originates in the distal nephron, also with an irregular cell arrangement, remarkable pleomorphism and hyperchromatic nuclei (Sircar et al., 2013).

Clinical diagnosis: Renal masses are diagnosed 50\% incidentally but some neoplasms due to their tumor 
behavior such as: tumor growth, metastatic disease, paraneoplastic syndrome or the degree of hematuria make evident the clinical suspicion of the patient. The classic triad of present symptoms is: pain in the lumbar fossa $(34 \%)$, presence of mass $(30 \%)$ and hematuria $(57 \%)$. Among, the paraneoplastic symptoms we have: weight loss (31\%), hypercalcemia (13\%), febrile syndrome $(20-33 \%)$, hepatic dysfunction (3-20\%), amyloidosis (1-5\%), anemia (20-40\%), polycythemia (1-8\%), hypertension $(25 \%)$.

\section{Pathological diagnosis}

TNM classification: The TNM system is recommended for clinical use as a prognostic value and for therapeutic use. Anatomical factors include: evidence of tumor, tumor size, venous invasion, invasion of the renal capsule, involvement of the adrenal and ganglionmetastasis and at a distance.

Anatopathological stages:

- $\quad$ Stage 1: T1 N0M0

- $\quad$ Stage 2: T2 NOMO

- $\quad$ Stage 3: T1 N1 Mo, T2 N1 Mo, T3 No Mo, T3 N1 M0

- $\quad$ Stage 4: T4NoM0, T4N1 Mo, T4N2 Mo, any T with any $\mathrm{NM} 1(10,12)$

Renal biopsy: Renal biopsy is used frequently for the diagnosis of renal tumors, for surveillance and ablation therapies. The biopsy determines a benign mass or the presence of malignancy in renal neoplasms as well as the histopathological type of renal cells and the degree of renal mass evaluated. The positive predictive value of the image data is quite high that a negative result of the biopsy does not alter the diagnostic-therapeutic attitude (Borghesi et al., 2015; Pilskog et al., 2017).

\section{Diagnostic imaging}

Renal score system: The SR is composed of five important features from the surgical point of view but only four add points for the calculation of nephrometry (Renal) on a scale of 1-3 points. The fifth item, $A$, adds a suffix (that is, a prior or $\mathrm{p}$ posterior) to the score, according to, the location of the lesion. The result of the summation classifies the tumors according to their surgical complexity in: low (between 4 and 6 points), moderate (between 7 and 9 points) and high (between 10 and 12 points) (Matsumoto et al., 2014).

\section{Prognostic factors}

Fuhrman nuclear grade: The Furhman nuclear grade is used as an independent prognostic value of survival, here, we consider the core size, the nuclear contour and the presence or absence of nucleolus. Next, classification: Grade 1: absent and basophilic nucleoli; Grade 2: conspicuous and eosinophilic nuclei at an increase of
X400; Grade 3: conspicuous and eosinophilic nuclei at an increase of X100; Grade 4: there is extreme nuclear polymorphism, multinucleated giant cells and sarcomatoid or rhabdoid differentiation.

Karnofsky index: The Karnofsky index is a functional scale that helps to predict the evolution and survival in cancer patients treated with chemotherapy, giving a score of $100 \%$ in normal patients without clinical signs and symptoms while of $90 \%$ any oncological patient capable of carrying a normal activity but with clinical signs and symptoms, a score of $80 \%$ in patients with normal activity but with effort, a karnofsky of $70 \%$ is when it is unable to perform any activity or active work, so, a karnofsky of $50 \%$ is when it is needed frequent medical assistance and finally if the oncological patient reaches a score of $30 \%$, he needs hospitalization.

Treatment: More than $50 \%$ of patients with early-stage renal cell carcinoma are cured but treatment for stage 4 disease is poor. The main treatment options for renal cell cancer are the following: Surgery (partial or radical nephrectomy), radiation therapy, immunotherapy, targeted molecular therapy.

Radical nephrectomy: Radical nephrectomy is the standard surgical procedure most commonly used for the treatment of localized renal cell carcinoma that involves the complete removal of Gerota's fascia and its contents including resection of kidney, perirenal fat and ipsilateral adrenal gland with or without lymph ipsilateral or ganglion dissection.

The objectives of the study were: To determine the degree of correlation between the parameters of histomorphological characterization and the morphoradiological findings objectified by computed axial tomography in patients older than 18 years who present malignant renal tumors treated at the Oncological Institute Dr. Juan Tanca Marengo, SOLCA-Guayaquil (Ecuador) in the period 2014-2016.

\section{MATERIALS AND METHODS}

Our study is descriptive, analytical and correlational observational in patients older than 18 years who present malignant renal masses objectified by the computed axial tomography and by the histopathological study that were attended in the Oncological Institute Dr. Juan Tanca Marengo, SOLCA during the period 2014-2016. All cases of the study population were analyzed.

Within the inclusion criteria were adult patients over 18 malignant years with computed axial tomography and histopathological study attended in the hospital. In contrast, the exclusion criteria were: 
- Patients with renal masses who have another radiological study different from computed tomography

- Patients whose age $\leq 18$ years and those who show benign renal masses regardless of age

- Patients whose medical records do not show the reports of tomographic or histopathological imaging studies

The study population obtained from the clinical histories of patients with malignant neoplasms treated at the SOLCA Institute, since, 2014-2016 were 225 cases, of which according to, the exclusion criteria, it was found that the statistical sample was of 74 patients that will be registered in the system (AS400) of SOLCA with the codes (CIE10: C64) that attended the outpatient service of the Urology Service.

The strategy of statistical analysis was to collect the information that was then tabulated in tables of Microsoft Excel 2016 and SSPS Statistics, according to, the study variables in which they were expressed with descriptive statistics, there is no intervention. The Chi-square tests were calculated, the statistical frequency was measured. A p $=0.05$ was taken as statistically significant. The variables used in our study are detailed in Table 1.

Table 1: Study variables

\begin{tabular}{|c|c|c|}
\hline Name/Definition of the variables & Types & Measurement levels \\
\hline \multicolumn{3}{|l|}{ Sex } \\
\hline Male, Female & Quantitative & Registry of clinical histories \\
\hline \multicolumn{3}{|l|}{ Age (years) } \\
\hline$\geq 18-30$ & Quantitative & Registry of clinical histories \\
\hline \multicolumn{3}{|l|}{$31-50$} \\
\hline \multicolumn{3}{|l|}{$51-70$} \\
\hline \multicolumn{3}{|l|}{$71-90$} \\
\hline \multicolumn{3}{|l|}{ Clinic } \\
\hline Asymptomatic & Quantitative & Registry of clinical histories \\
\hline \multicolumn{3}{|l|}{ Pain in the lumbar fossa } \\
\hline \multicolumn{3}{|l|}{ Macroscopic hematuria } \\
\hline \multicolumn{3}{|l|}{ Palpable mass } \\
\hline \multicolumn{3}{|l|}{ Weight loss } \\
\hline \multicolumn{3}{|l|}{ Pain in the costal arch } \\
\hline \multicolumn{3}{|l|}{ Pain in flanks } \\
\hline \multicolumn{3}{|l|}{ Cough+dyspnea } \\
\hline \multirow{2}{*}{\multicolumn{3}{|c|}{$\begin{array}{l}\text { Diffuse abdominal pain } \\
\text { Histopathological report }\end{array}$}} \\
\hline & & \\
\hline Malignant epithelial,mesenchymal and urothelial origin renal tumors & Quantitative & Kidney biopsy report and/or exeresis \\
\hline \multicolumn{3}{|l|}{ Radiological study by TAC (score renal) } \\
\hline Tumor size & Quantitative & Record of pre-surgical radiological reports \\
\hline \multicolumn{3}{|l|}{ Exophytic/endophytic properties } \\
\hline \multicolumn{3}{|l|}{ Proximity of the tumor to the collecting or sinus system } \\
\hline \multicolumn{3}{|l|}{ Previous/later } \\
\hline \multicolumn{3}{|l|}{ Location relative to the polar lines } \\
\hline \multicolumn{3}{|l|}{ Fuhrman scale } \\
\hline 1 & Quantitative & Renal biopsy report and/or exeresis \\
\hline \multicolumn{3}{|r|}{ 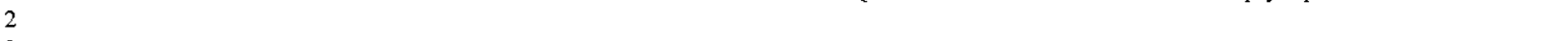 } \\
\hline \multicolumn{3}{|l|}{3} \\
\hline \multicolumn{3}{|l|}{4} \\
\hline Karnofsky index (\%) & & \\
\hline 100 & Quantitative & Registry of clinical histories \\
\hline 90 & & \\
\hline 80 & & \\
\hline 60 & & \\
\hline 50 & & \\
\hline Tumor invasions & & \\
\hline Lymphovascular & Quantitative & Renal biopsy report and/or exeresistregistry \\
\hline Perirenal fat & & of radiological reports \\
\hline Fascia of Gerota & & \\
\hline Renal sinus & & \\
\hline Renal vein & & \\
\hline Vena cava superior and inferior & & \\
\hline Renal capsule & & \\
\hline No. of lymph nodes & & \\
\hline Two & Quantitative & Renal biopsy report and/or exeresis \\
\hline Three & & \\
\hline Four & & \\
\hline Five & & \\
\hline
\end{tabular}


Res. J. Pharmacol., 13 (3): 35-41, 2019

\begin{tabular}{|c|c|c|}
\hline Name/Definition of the variables & Types & Measurement levels \\
\hline \\
\hline Ten & & \\
\hline \multicolumn{3}{|l|}{ Nodular localization } \\
\hline Paracaves & Qualitative & Register of radiological reports \\
\hline \multicolumn{3}{|l|}{ Para-aortic } \\
\hline \multicolumn{3}{|l|}{ Pulmonary } \\
\hline \multicolumn{3}{|l|}{ Retroperitoneal } \\
\hline \multicolumn{3}{|l|}{ Paracavos+pulmonary } \\
\hline \multirow{2}{*}{\multicolumn{3}{|c|}{$\begin{array}{l}\text { Paracaves+aortic } \\
\text { Distant metastasis }\end{array}$}} \\
\hline & \multicolumn{2}{|c|}{ Distant metastasis } \\
\hline Liver & Qualitative & Renal biopsy report and/or exeresis+registry \\
\hline Lung & & of radiological reports \\
\hline \multicolumn{3}{|l|}{ Liver+lung } \\
\hline \multicolumn{3}{|l|}{ Retroperitoneum } \\
\hline \multicolumn{3}{|l|}{ Scapula, sternum } \\
\hline \multicolumn{3}{|l|}{ Tomographic density } \\
\hline Hypodensa & Qualitative & Register of radiological reports \\
\hline \multicolumn{3}{|l|}{ Hyperdensa } \\
\hline \multicolumn{3}{|l|}{ Radiopathological correlation } \\
\hline Yes & Qualitative & Analysis \\
\hline \multicolumn{3}{|l|}{ No } \\
\hline \multicolumn{3}{|l|}{ Local stage } \\
\hline Primary tumor & Qualitative & TNM scale \\
\hline \multicolumn{3}{|l|}{ Regional lymph nodes metastasis ata distance } \\
\hline Surgical complexity & & \\
\hline Partial nephrectomy & Qualitative & Low \\
\hline Two; radical nephrectomy & & Moderate \\
\hline Medical and palliative & & High \\
\hline
\end{tabular}

\section{RESULTS AND DISCUSSION}

In the period studied, we found 225 patients with renal masses, of them 25 were minors, 40 did not have Tac, 6 did not have microscopy reports, 80 did not have both criteria, leaving a sample of $74(33 \%)$ patients with kidney cancer that we include in this research.

Sex: Of the sample studied $(\mathrm{n}=74)$ in relation to sex, the variable was distributed equitably $50 \%$ (37 cases) for each gender.

Clinical symptomatology: Within the clinical findings were asymptomatic (23\%). Pain in the lumbar fossa was considered as the most prevalent symptom characteristic in $(22 \%)$ cases. Macroscopic hematuria was reported in (17\%) patients, while in $26 \%$ the presence of palpable mass was evidenced in the patients. In a few cases $(5 \%)$ weight loss was detailed. Other less specific symptoms were pain in the costal arch (1\%), pain in the flanks (3\%), cough + dyspnea $(5 \%)$ and diffuse abdominal pain $(1 \%)$ of the cases reported.

In a comparative study in Korea in 2015 , it was documented that most of the cases are discovered incidentally (76.9\%) and in second place is hematuria (10.4\%) and lumbar pain (8.2\%) (Byun et al., 2016).

In our study, it was possible to demonstrate that $30 \%$ of the renal masses were malignant which disagrees with the study in Mexico, where $7 \%$ presented malignant renal masses, this may be due to the fact that in our environment. The lesion is presumed to be benign and is usually treated at its base hospitals while if malignancy is suspected it is more likely that it is referred to the oncological hospital.

According to, an observational, retrospective study conducted at the Oncology Urology Clinic, the sex most affected was men in relation $(2: 1)$. In our research, we found a 1:1 ratio for both sexes.

Age: With regard to the age group, the most prevalent was between $51-70$ years with $65 \%$ of the patients with an average age of 59 years, a result very similar to that obtained by the Committee of the Japanese Association of Urology which describes an average of 63 (59-95 years), reinforcing the idea that this type of cancer is classically late (60 years and older) (Costilla-Montero et al., 2011).

Karnofsky index: The study details that only 4 patients (5.41\%) had a Karnofsky of $100 \%$ (totally asymptomatic). Being the Karnofsky of $80 \%$ (presence of some clinical signs and symptoms) the most prevalent as in the study conducted by the Instituto Nacional de Rinon y Trasplante de.

Karnofsky was not reported in 21 cases (28\%). A $21.62 \%$ (16 cases) have a karnofsky of $90 \%$ (able to develop a normal daily life). In 3 cases (4\%) there was evidence of a $60 \%$ Karnofsky (patients requiring occasional care to meet most of their needs) and in one case $(1 \%)$ required frequent medical care (Karnofsky $50 \%)$. 


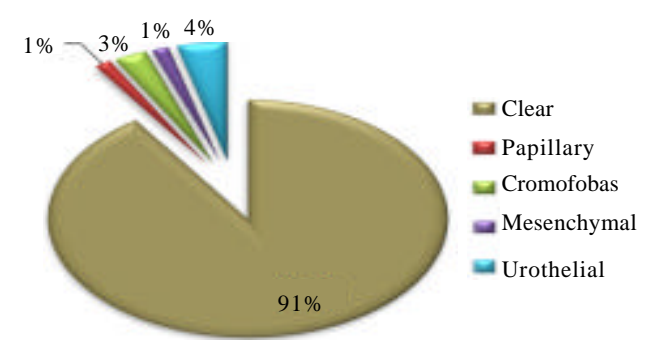

Fig. 2: Cell type

Fuhrman scale: According to the Fuhrman scale, grade 2 represented almost $60 \%$ of the cases, followed by grade $3(19 \%)$, grade $1(14 \%)$ and $5 \%$ for grade 4 . In the study of the Instituto Nacional de cancer (2018), the most common nuclear grade of renal carcinoma was also grade $2(51.8 \%)$, followed by grade $3(37.4 \%)$, grade $4(6.1 \%)$ and grade 1 $(4.8 \%)$.

Cell type and infiltration to the renal capsule: The following results were estimated in the pathology: The renal tumor infiltrates the capsule by $31 \%$ (23), similar to that obtained in a study in Korea (2013) with $25 \%$ of the cases (Hwang et al., 2013).

In contrast in 50 cases (68\%) it is not observed that it exceeds the renal capsule. In 1 sample there was no report. The most frequent histological variant was that of clear cells with 67 cases $(91 \%)$, the remaining being the papillary $(4 \%)$, the chromophobe $(3 \%)$ and the mesenchymal type (sarcoma, $2 \%$ ). According to, a study conducted at the Bundang University Hospital (2015), the most common histological subtype is renal clear cell carcinoma in $85.2 \%$ of cases, chromophobe cell carcinoma (6.4\%) and papillary carcinoma (1\%) (Byun et al., 2016) (Fig. 2).

Number of lymphoid nodules: In $38 \%$ of patients, Histopathology reports showed infiltration to lymph nodes, most of them with 4 affected lymph nodes (14\%). On the other hand, the study by Molinette University Hospital (2010) showed lymph node invasion in most patients (83\%) with more than 12 lymph nodes affected (45\%) (Frunza et al., 2012). This situation may be due to the fact that during the macroscopic evaluation, the pathologist does not perform adequate ganglionic isolation of the surgical specimen or that the surgeon does not remove the lymphoid nodes in their entirety.

Location of lymphoid nodules: The $31 \%$ of the patients presented infiltration of kidney cancer to the lymph nodes. Among them were the following: paracaval (9\%), para-aortic ( $9 \%)$. The minority of them (3\%) were located in the pulmonary and retroperitoneal nodules. According to, a retrospective study conducted in Spain by Balaguer et al. reviewed infiltration to pulmonary lymph nodes in $40 \%$ of patients.

Status of the tumor: The majority of the tumor invades the upper portion of the kidney (39;53\%). In 19 cases $(26 \%)$ it affects the lower part of the kidney leaving $5 \%$ in the medial part of the kidney. According to, a retrospective study on renal nephrometry at Hokkaido University Hospital by Matsumoto et al. (2014) indicated that the renal masses are located in the lower part of the kidney $(59.6 \%)$, superior $(29.8 \%)$ and middle portion $(10.6 \%)$ disagreeing with our study because most of the Urologists in Ecuador do not use the kidney score methodology.

TNM staging: Within tumor staging or TNM, stage 1 represents primary tumors without affected lymph nodes or metastases corresponding to $(35 \%)$ of the cases. Stage 2 represents tumors larger than $7 \mathrm{~cm}$ but limiting to the kidney that corresponded to $(18 \%)$ and stage 3 are the tumors corresponding to $\mathrm{T} 1-\mathrm{T} 3$ plus invasion to ganglia that represent $(18 \%)$. Finally, stage 4 are tumors that invade Gerota's fascia, lymph nodes and metastases that correspond to $27 \%$.

Metastasis at a distance: The distant metastasis corresponds to $26 \%$ and were observed in the liver and lungs $(16 \%)$. In $3 \%$ there was infiltration to the peritoneum. The $1 \%$ metastasized to the adrenal glands, retro peritoneum, scapula and sternum. According to, a prospective cohort study conducted at Haukeland University Hospital, Bergen indicated that the most frequent site of metastasis was in the lungs $(76.1 \%)$, liver $(8.7 \%)$ and bones $(34.8 \%)$ by which disagrees with our study due to the lack of pathological reports made at sites of metastasis (Pilskog et al., 2017).

Procedures and surgical complexity: According to, the parameters used in the renal score, it is possible to know the degree of surgical complexity in which it is categorized into: low (4-6) in 46\%, moderate (7-9) in $38 \%$ and high (10-12) at 16\%. Regarding the procedures performed, radical nephrectomy was observed $(54.73 \%)$ cases, followed by partial nephrectomy in $22 \%$ and renal biopsy in $4 \%$ of them. The Chi-square between the partial and radical nephrectomy according to, the degree of surgical complexity was $\mathrm{p}=0.00$ which indicates that the study is significant, so, there is no correlation between the urologist and the radiologist. 
Tomographic density: The images of the renal masses showed that the majority have a minimum density of $74 \%$ (55). In contrast in $26 \%$ (19) cases reported being hyperdense images. In all the reports about the renal masses they are heterogeneous and the uptake of the contrast medium is intense.

In terms of lymphatic vascular invasions $(31,32 \%)$, perirenal fat $(24 \%)$, Gerota's fascia $(5 \%)$, renal sinus $(25.6 \%)$, renal vein $(18.9 \%)$, cava superior vein $(2.7 \%)$, cava inferior vain $(6.7 \%)$.

Characteristics of the tumor: The average size of the renal masses in the microscopy was 7.43 and in the images 7.48 with a range that goes from $2-17 \mathrm{~cm}$. Therefore, it is not significant in our study. According to, a retrospective study conducted at the Central University Hospital of Asturias it was observed that the mean tumor size was $7.17 \pm 3.4(2-20 \mathrm{~cm})$ (Jalon et al., 2007).

Intratumoral necrosis was observed in $30(40.5 \%)$ cases in the anatopathological report and $27(36 \%)$ in the tomography report considered as not significant in the study.

Lymph node metastasis: We found $20(25.6 \%)$ cases reported in the report of the biopsies and $21(27 \%)$ reported cases in the reports of the tomography images. The Chi-squared test was performed where the result was $p=0.93$ where the study was not significant.

Finally, there is an equitable proportion according to the laterality of the tumor, both right and left (50:50). According to, a study carried out in Urological Oncology (2017) it was shown that laterality was equal on both sides $(49.4,50 \%)$, so, if it correlates with our study (Ryu et al., 2017).

\section{CONCLUSION}

After 59 years of age, there is an increased risk of kidney cancer. In most cases, renal cell carcinoma is discovered by independent imaging of renal pathology. Renal clear cell carcinoma was the most frequent histological type with a grade 2 Fuhrman present in the majority of cases. It has been shown that renal tumors can infiltrate the renal capsule, perirenal fat, Gerota's fascia, renal sinus, renal vein, vena cava and the lymphovascular system. The presence of tumor necrosis has been detected in patients with renal masses greater than $7 \mathrm{~cm}$. The mean tumor size was $7.17(2-20) \mathrm{cm}$. The majority of patients diagnosed are in stage 1-3. The degree of surgical complexity indicates that there is no correlation $(\mathrm{p}=0.00)$ between the imaging report and the surgical evaluation of urologists.

\section{REFERENCES}

Borghesi, M., E. Brunocilla, A. Volpe, H. Dababneh and C.V. Pultrone et al., 2015. Active surveillance for clinically localized renal tumors: An updated review of current indications and clinical outcomes. Intl. J. Urol., 22: 432-438.

Byun, S.S., S.K. Hong, S. Lee, H.R. Kook and E. Lee et al., 2016. The establishment of KORCC (Korean Renal Cell Carcinoma) database. Investig. Clin. Urol., 57: 50-57.

Costilla-Montero, A., B. Guadarrama-Benitez, M.A. Aragon-Castro, R. Gutierrez-Rosales and $\mathrm{O}$. Morales-Ordaz et al., 2011. Oncocitoma renal incidental. Rev. Mex. Urol., 71: 360-362.

Frunza, A., L.G. Florez, M.M. Camaces, G.G. Alvarez and C.M. Alonso et al., 2012. 338. Correlation between lymph node status and tumour regression after neoadyuvant chemoradiation in locally advanced rectal cancer. Eur. J. Surg. Oncol., 38: 836-837.

Hwang, E.C., H.S. Yu and D. Kwon, 2013. Small renal masses: Surgery or surveillance. Korean J. Urol., 54: 283-288.

Jalon, A.M., M.M. Alvarez, J.G. Fernandez, J.B. Martin and F.G. Martinez et al., 2007. Renal cell carcinoma: Prognostic factors and staging. Arch. Esp. Urol., 60: 125-136.

Karam, J.A., X.Y. Zhang, P. Tamboli, V. Margulis and H. Wang et al., 2011. Development and characterization of clinically relevant tumor models from patients with renal cell carcinoma. Eur. Urol., 59: 619-628.

Matsumoto, R., T. Abe, N. Shinohara, S. Murai and S. Maruyama et al., 2014. RENAL nephrometry score is a predictive factor for the annual growth rate of renal mass. Intl. J. Urol., 21: 549-552.

Pilskog, M., C. Beisland, L.A. Akslen, L. Bostad and A. Haug et al., 2017. Predictive value of C-reactive protein in patients treated with sunitinib for metastatic clear cell renal cell carcinoma. BMC. Urol., 17: 1-11.

Ryu, H., B. Song, J. Hwang, S.K. Hong and S.S. Byun et al., 2017. Pelvic lymph node metastases in prostate cancer: Preoperative detection with dynamic contrast-enhanced magnetic resonance imaging compared with postoperative pathologic result of pelvic lymph node dissection. Korean J. Urol. Oncol., 15: $158-164$.

Sircar, K., P. Rao, E. Jonasch, F.A. Monzon and P. Tamboli, 2013. Contemporary approach to diagnosis and classification of renal cell carcinoma with mixed histologic features. Chinese J. Cancer, 32: 303-311.

Villaverde, R.M., L.V. Leon, B.M.A. Martinez and E.R. Gonzalez, 2017. Cancer renal. Med. Programa Formacion Med. Continuada Acreditado, 12: 1947-1954. 